\title{
DESENVOLVIMENTO E AVALIAÇÃO DE UMA COLUNA DE LEITO ROTATIVO NA ABSORÇÃO DE DIÓXIDO DE CARBONO COM HIDRÓXIDO DE SÓDIO
}

\author{
K. S. G. C. OLIVEIRA ${ }^{1}$, C. C. de CARVALHO ${ }^{1}$, F. S. VIEIRA ${ }^{1}$, L. M. SANTOS ${ }^{1}$ e J. D. A. \\ BELLIDO $^{1}$
}

${ }^{1}$ Universidade Federal de São João del-Rei, Departamento de Engenharia Química E-mail para contato: kaiquesg.eq@ gmail.com

\begin{abstract}
RESUMO - As colunas de recheio rotativo, denominadas Higee, permitem o aumento da capacidade e eficiência de um processo, comparado a uma coluna convencional. A principal proposta deste trabalho é desenvolver e a avaliar uma coluna de leito estruturado rotativo para a absorção de gás carbônico por uma solução de hidróxido de sódio $1 \mathrm{M}$, a partir da eficiência de captura de $\mathrm{CO}_{2}$, da área interfacial efetiva $\left(\mathrm{a}_{\mathrm{e}}\right)$ e do coeficiente volumétrico gasoso de transferência de massa $\left(\mathrm{k}_{\mathrm{G}} \mathrm{A}\right)$, aplicando a tecnologia Higee. Assim como, avaliar a influência da velocidade de rotação e das vazões de gás e líquido. A absorção de $\mathrm{CO}_{2}$ na solução de $\mathrm{NaOH}$ foi determinada a partir da titulação com $\mathrm{HNO}_{3} 1 \mathrm{M}$. Foram testadas velocidades de rotação de 300, 1050 e $1800 \mathrm{rpm}$, vazões de $\mathrm{CO}_{2}$ de 4, 6 e 8 L. min $^{-1}$ e vazões de $\mathrm{NaOH}$ de 0,5, 0,75 e 1 L. min $^{-1}$. Os resultados obtidos confirmam que o aumento da velocidade de rotação, aumentou a eficiência, a ae, $o$ $\mathrm{k}_{\mathrm{G}} \mathrm{A}$, e, consequentemente, o contato entre as fases. A proporção direta também se observou entre a vazão de gás e os valores de $a_{e}$ e $k_{G} A$. As maiores eficiências foram obtidas nas menores vazões de $\mathrm{CO}_{2}$ e $\mathrm{NaOH}, 4 \mathrm{~L} \cdot \mathrm{min}^{-1}$ e $0,5 \mathrm{~L} \cdot \mathrm{min}^{-1}$ respectivamente, e na maior velocidade de rotação, $1800 \mathrm{rpm}$.
\end{abstract}

\section{INTRODUÇÃO}

O conceito de Intensificação de Processos surgiu da possibilidade de reduzir os custos totais de operação em uma planta industrial por meio da redução de seu tamanho, com isso tem-se o aumento da produtividade e da qualidade dos produtos. Nesse contexto, associa-se a tecnologia Higee, oriunda do termo em inglês "high g", que significa alta gravidade, desenvolvida por Ramshaw e Mallinson (1981). Ela utiliza a força centrífuga para intensificar a transferência de massa interfacial gás-líquido, reduzindo o volume do equipamento em até duas vezes em comparação com o modelo convencional (Joel et al., 2014).

Durante as últimas três décadas, colunas rotativas ou RPB (do inglês, Rotating Packed Bed) foram empregados para obter absorção (Zhang et al., 2011), dessorção (Majeed et al., 2007), destilação (Luo et al., 2012), desvolatilização de polímeros (Chen et al., 2010), precipitação reativa (Chen et al., 2000), produção de nanopartículas (Sun et al., 2011), entre outros.

A demanda de energia associada ao aumento do uso de combustíveis fósseis, aumentou consideravelmente as concentrações de $\mathrm{CO}_{2}$ na atmosfera. Muitos estudos avaliaram a viabilidade 
de captura de $\mathrm{CO}_{2}$ utilizando o processo de absorção em colunas rotativas com soluções aquosas de MEA (Joel, 2014), etanolamina (Yeh; Pennlie, 2001) ou NaOH (Liu, 2015), por exemplo.

De modo a trazer subsídios ao projeto de novas colunas de absorção ou de modificações de colunas já existentes, esse trabalho teve o objetivo de construir e analisar o desempenho da coluna de absorção com recheio estruturado e rotativo, atuando na mistura binária $\mathrm{NaOH} / \mathrm{CO}_{2}$. Para tanto, foram determinados o coeficiente de transferência de massa para a fase líquida, além da influência da velocidade de rotação, das vazões de gás e líquido na eficiência de remoção de $\mathrm{CO}_{2}$.

\section{MATERIAIS E METODOLOGIA}

\subsection{Montagem e operação da coluna rotativa}

A unidade Higee foi construída por rotor com recheio estruturado e auxiliares, como descrito na Figura 1. A solução de $\mathrm{NaOH} 1 \mathrm{M}$ é alimentada através de um distribuidor pelo interior da coluna e flui radialmente para fora, como gotículas ou filetes através do recheio, por meio da força centrífuga. Enquanto isso, a mistura gasosa (ar e $10 \%$ de $\mathrm{CO}_{2}$ ) entra no interior do rotor, pelo lado externo, e flui radialmente para dentro por um gradiente de pressão. A coleta do líquido é feita pela parte de baixo.

Figura 1 - Diagrama da montagem experimental.

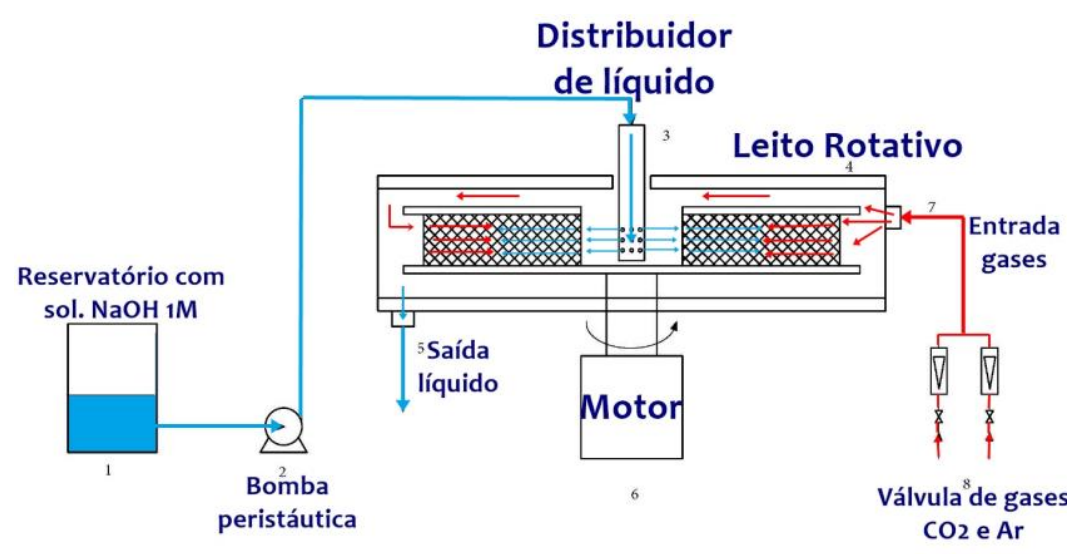

\subsection{Planejamento Experimental}

Utilizou-se o design de experimentos fracionado (Tabela 1) para avaliar a influência de três parâmetros no processo: vazão de solução de hidróxido, a rotação do motor e a vazão da mistura gasosa, totalizando sete experimentos.

Tabela 1 - Variáveis e níveis de estudo.

\begin{tabular}{|c|c|c|c|}
\hline Níveis & $\begin{array}{c}\text { Vazão de } \\
\mathrm{NaOH} 1 \mathrm{M} \\
\left(\mathrm{L} \cdot \mathrm{min}^{-1}\right) \\
\end{array}$ & $\begin{array}{l}\text { Rotação do } \\
\text { motor (rpm) }\end{array}$ & $\begin{array}{l}\text { Vazão de } \mathrm{CO}_{2} \\
\quad\left(\text { L. } \text { min }^{-1}\right)\end{array}$ \\
\hline $\begin{array}{l}-1 \\
\end{array}$ & 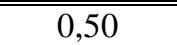 & 300 & 44,0 \\
\hline 0 & 0,75 & 1050 & 6,0 \\
\hline 1 & 1,00 & 1800 & 8,0 \\
\hline
\end{tabular}




\subsection{Eficiência de captura de $\mathrm{CO}_{2}$}

A eficiência de captura do $\mathrm{CO}_{2}$ foi calculada pela Equação 1. A concentração inicial de $\mathrm{CO}_{2}\left(\mathrm{C}_{\mathrm{i}}\right)$ foi calculada por meio da vazão de mistura $\mathrm{CO}_{2}$ e ar da alimentação. A concentração final $\left(\mathrm{C}_{\mathrm{f}}\right)$ foi determinada indiretamente, por meio da titulação da solução de $\mathrm{NaOH}$ com $\mathrm{HNO}_{3} 1 \mathrm{M}$, utilizando a fenolftaleína como indicador.

$$
E(\%)=\frac{C_{i}-C_{f}}{C_{i}} * 100
$$

\section{RESULTADOS E DISCUSSÃO}

\subsection{Principais efeitos das variáveis na eficiência}

A Figura 2a apresenta o efeito da velocidade de rotação na eficiência de captura de $\mathrm{CO}_{2}$, para cada vazão de $\mathrm{CO}_{2}$ testada, na concentração de $10 \%$ v/v. Conforme esperado, o aumento da velocidade de rotação proporcionou um aumento na eficiência de captura de $\mathrm{CO}_{2}$, pelo fato da aceleração centrífuga fornecer um filme estagnado de fina espessura junto à interface gáslíquido, conduzindo a uma maior transferência de massa, de acordo com a teoria da penetração, desenvolvida por Higbie (1935). Verifica-se também que na menor vazão de gás (4 $\mathrm{L} \mathrm{min}^{-1}$ de $\mathrm{CO}_{2}$ ), obteve-se a maior eficiência na captura do $\mathrm{CO}_{2}$. O aumento na vazão do gás resulta em um tempo menor de contato entre as fases gás-líquido, diminuindo assim a transferência de massa. Tendências semelhantes foram encontradas por Lin e Chen (2011) ao estudar a absorção de $\mathrm{CO}_{2}$ pela solução de $\mathrm{NaOH}$ no RPB de fluxo cruzado com recheio estruturado.

Figura 2 - Efeito da velocidade de rotação na eficiência para cada vazão testada de: (a) $\mathrm{CO}_{2} \mathrm{e}$ (b) $\mathrm{NaOH}$.
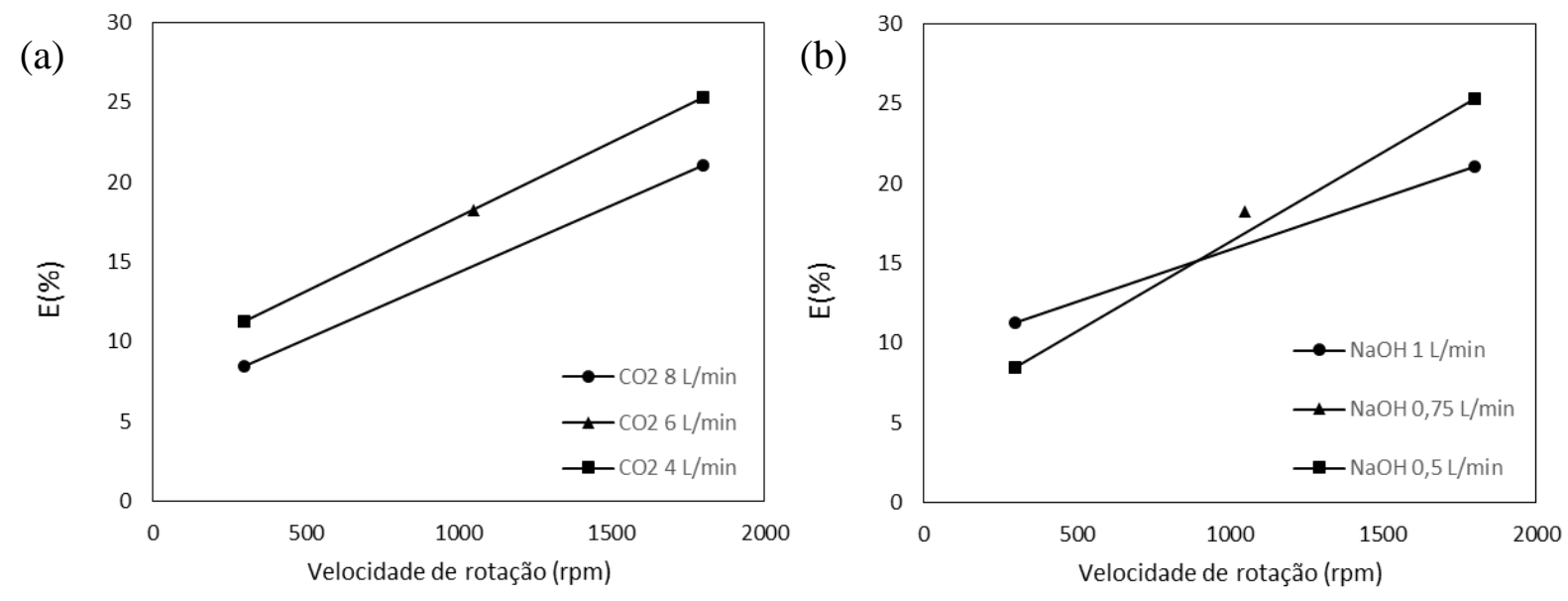

Pela Figura 2b, verificou-se que a melhor eficiência foi obtida trabalhando-se com a velocidade de rotação e vazão de $\mathrm{NaOH}$ inversamente proporcionais. É interessante prever, mesmo sem os testes, que é possível obter a mesma eficiência, para uma velocidade de rotação de aproximadamente $900 \mathrm{rpm}$, usando tanto uma vazão de 0,5 quanto de $1 \mathrm{~L}^{\mathrm{min}}{ }^{-1}$ do hidróxido. 


\subsection{Efeito padronizado das variáveis na eficiência}

É possível concluir com a Figura 3, que a rotação é a variável preditora mais significativa na eficiência da captura de $\mathrm{CO}_{2}$ pela solução de $\mathrm{NaOH} 1 \mathrm{M}$, na coluna de leito rotativo.

Figura 3 - Efeito padronizado das variáveis na eficiência.

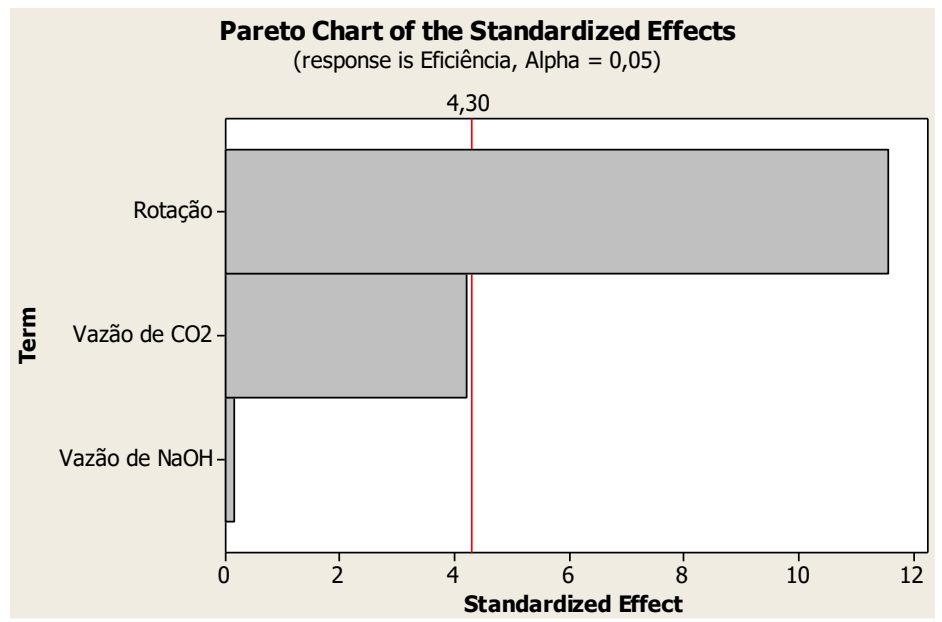

\subsection{Efeito da velocidade de rotação e da vazão de $\mathrm{CO}_{2}$ sobre o $\mathbf{k}_{\mathrm{G}} \mathrm{A}$ e $\mathrm{a}_{\mathrm{e}}$}

A Figura 4 mostra a relação entre a velocidade de rotação com a área interfacial efetiva $\left(\mathrm{a}_{\mathrm{e}}\right)$ e o coeficiente volumétrico gasoso de transferência de massa $\left(\mathrm{k}_{\mathrm{G}} \mathrm{A}\right)$, variando-se a vazão de $\mathrm{CO}_{2}$. Aumentando-se a velocidade de rotação, aumenta-se a força de cisalhamento do líquido pelo recheio, melhorando a dispersão do líquido e consequentemente aumentando a $\mathrm{a}_{\mathrm{e}} \mathrm{e}$ o $\mathrm{k}_{\mathrm{G}} \mathrm{A}$. O aumento da vazão do $\mathrm{CO}_{2}$ também provoca um aumento da $\mathrm{a}_{\mathrm{e}} \mathrm{e}$ do $\mathrm{k}_{\mathrm{G}} \mathrm{A}$, devido ao aumento da velocidade radial do mesmo, conduzindo a uma maior perturbação das fases gás-líquido e com isso, uma melhor dispersão do líquido e um aumento da interface gáslíquido (Yang et al., 2011).

Figura 4 - Efeito da velocidade de rotação (a) na $\mathrm{a}_{\mathrm{e}} \mathrm{e}(\mathrm{b})$ no $\mathrm{k}_{\mathrm{G}} \mathrm{A}$, variando a vazão de $\mathrm{CO}_{2}$.
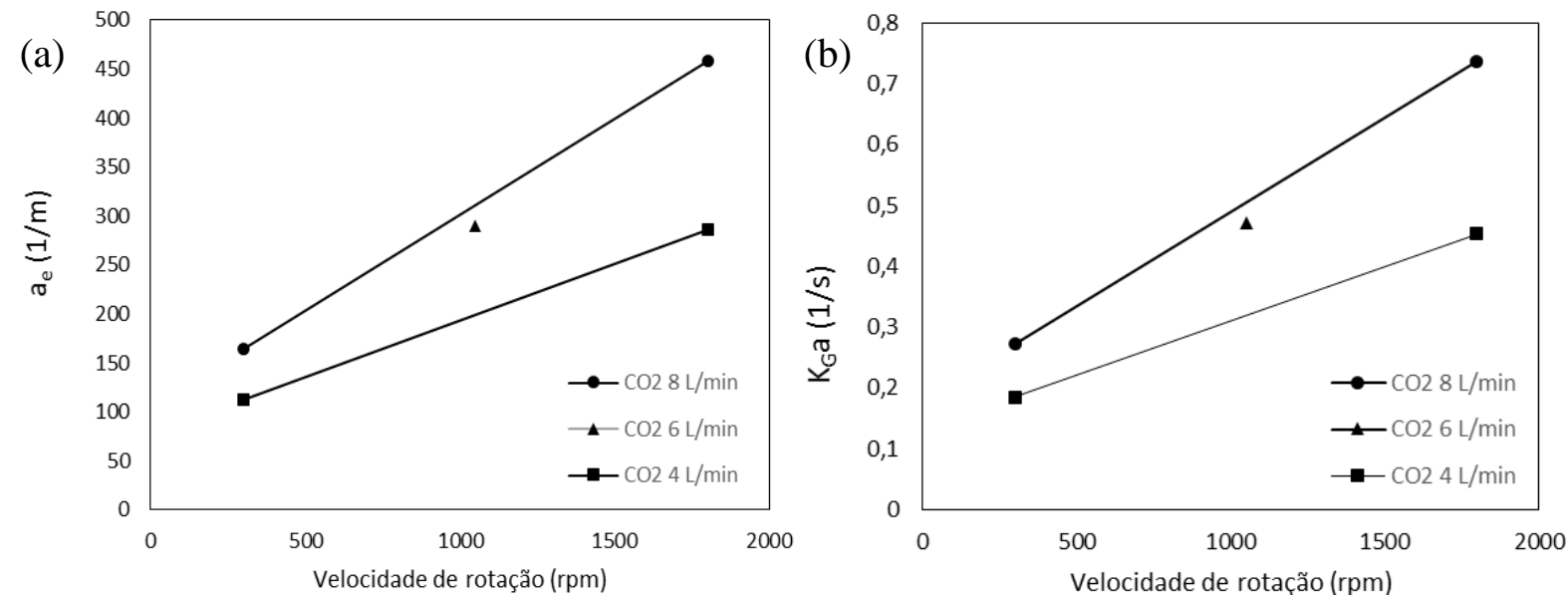
A Figura 5 mostra a relação entre a velocidade de rotação com a $\mathrm{a}_{\mathrm{e}} \mathrm{e}$ o k $\mathrm{k}_{\mathrm{G}} \mathrm{A}$, variandose a vazão de $\mathrm{NaOH}$. Para a menor rotação são obtidos valores menores de $\mathrm{a}_{\mathrm{e}}$, e $\mathrm{k}_{\mathrm{G}} \mathrm{A}$, quando se utilizou a menor vazão de $\mathrm{NaOH}$. Esse efeito pode ser explicado pela insuficiência da rotação em dispersar o líquido que entra no recheio, formando filmes líquidos mais espessos, dificultando a entrada do gás e diminuindo a interface gás-líquido. Já para a maior rotação, são obtidos valores maiores de $\mathrm{a}_{\mathrm{e}}$, e $\mathrm{k}_{\mathrm{G}} \mathrm{A}$, quando se tem a maior vazão de $\mathrm{NaOH}$. Isso ocorre porque a velocidade de rotação consegue dispersar melhor o líquido e o aumento da velocidade do líquido promove a formação de filmes líquidos mais finos e a fração da embalagem molhada também aumenta (Rajan et al., 2011).

Figura 5 - Efeito da velocidade de rotação (a) na ae e (b) no $\mathrm{k}_{\mathrm{G}} \mathrm{A}$, variando a vazão de $\mathrm{CO}_{2}$, variando a vazão de $\mathrm{NaOH}$.
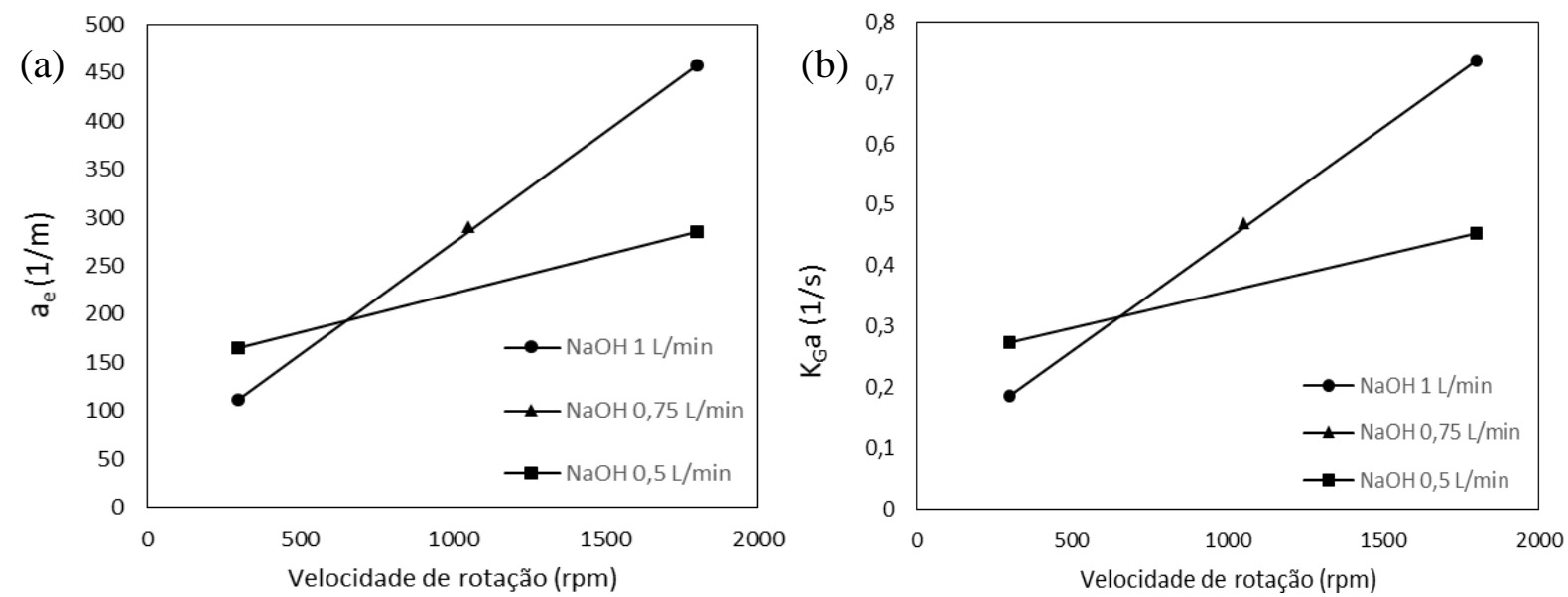

\section{CONCLUSÃO}

O uso da coluna rotativa com recheio estruturado mostra-se com uma importante aplicação industrial na redução do volume de equipamentos e a redução dos custos. Este trabalho propôs a construção e a avaliação de uma coluna de leito estruturado e rotativo para a absorção de gás carbônico por hidróxido de sódio. Os resultados foram avaliados em relação à eficiência de captura de $\mathrm{CO}_{2}$, à área interfacial efetiva $\left(\mathrm{a}_{\mathrm{e}}\right)$, e ao coeficiente volumétrico gasoso de transferência de massa $\left(\mathrm{k}_{\mathrm{G}} \mathrm{A}\right)$, utilizando planejamento experimental. Os resultados indicaram que o aumento da velocidade de rotação, acarretou no aumento da eficiência, da $\mathrm{a}_{\mathrm{e}}$ e do $\mathrm{k}_{\mathrm{G}} \mathrm{A}$. Conclui também que quanto proporcionalidade direta entre a vazão de gás, com os valores de $a_{e}$ e $k_{G} A$. As condições de operação testadas, que ofereceram a maior eficiência, foram as menores vazões de $\mathrm{CO}_{2}$ e $\mathrm{NaOH}, 4 \mathrm{~L} \cdot \mathrm{min}^{-1}$ e $0,5 \mathrm{~L} \cdot \mathrm{min}^{-1}$, respectivamente, e a maior velocidade de rotação, $1800 \mathrm{rpm}$. Por isso, sugere-se modificações no recheio para aumentar a eficiência e para que seja possível trabalhar com maiores vazões de $\mathrm{CO}_{2}$.

\section{REFERÊNCIAS}

CHEN, J. F.; GAO, H.; ZOU, H. K.; CHU, G. W.; ZHANG, L.; SHAO, L.; XIANG, Y.; WU, Y. X. Cationic Polymerization in Rotating Packed Bed Reactor: Experimental and Modeling. AIChE J. v. 56, p. 1053, 2010. 
CHEN, J. F.; WANG, Y. H.; GUO, F.; WANG, X. M.; ZHENG, C. Synthesis of Nanoparticles with Novel Technology: High-gravity Reactive Precipitation. Ind. Eng. Chem. Res., v. 39, p. 948, 2000.

HIGBIE, R. The rate of absorption of a pure gas into a still liquid during short periods of exposure. J. Amer. Inst. Chem. Eng., v. 31, p. 365-390, 1935.

JOEL, A. S.; WANG, M.; RAMSHAW, C.; OKO, E. Process analysis of intensified absorber for post-combustion $\mathrm{CO}_{2}$ capture through modelling and simulation. Int J Gas Control, v. 21, p. 91-100, 2014.

LIN, C. C.; CHEN, B. C. Carbon dioxide absorption into $\mathrm{NaOH}$ solution in a cross-flow rotating packed bed. J. Ind. Eng. Chem., v. 13, p. 1083-1090, 2011.

LIU, Y.; GU, D.; XU, C; QI, G.; JIAO, W. Mass transfer characteristics in a rotating packed bedwith split packing. Chin. J. of Chem. Eng., v. 23, p. 868-872, 2015.

LUO, Y.; CHU, G. W.; ZOU, H. K.; XIANG, Y.; SHAO, L.; CHEN, J. F. Characteristics of a two-stage counter-current rotating packed bed for continuous distillation. Chem. Eng. Process., v. 52, p. 55, 2012.

MAJEED, S. J.; ROCHELLE, G.; EIMER, D.; RAMSHAW, C. Carbon Dioxide Absorption and Desorption in Aqueous Mono Ethanol Amine Solutions in a Rotating Packed Bed. Ind. Eng. Chem. Res., v. 46, p. 2823, 2007.

RAJAN S.; KUMAR M.; ANSARI M. J.; RAO D. P.; KAISTHA N. Limiting gas-liquid flows and mass transfer in a novel rotating packed bed (HiGee), Ind. Eng. Chem. Res., v. 50, p. 986-997, 2011.

RAMSHAW, C.; MALIINSON, R. H. Mass transfer process. U.S. Patent 4.283.255, 1981.

SUN, B. C.; WANG, X. M.; CHEN, J. M.; CHU, G. W.; CHEN, J. F.; SHAO, L. Synthesis of Nano-CaCO 3 by Simultaneous Absorption of $\mathrm{CO}_{2}$ and $\mathrm{NH}_{3}$ into $\mathrm{CaCl}_{2}$ Solution in a Rotating Packed Bed. Chem. Eng. J., v. 168, p. 731, 2011.

YANG, K.; CHU, G. W; ZOU, H. K.; SUN, B. C.; SHAO, L.; CHEN, J. F. Determination of the effective interfacial area in rotating packed bed. Chem. Eng. J. v. 168, p. 13771382, 2011.

YEH, J. T., PENNLINE, H. W. Study of $\mathrm{CO}_{2}$ Absorption and Desorption in a Packed Column. U.S. DOE/ NETL, Kevin P. Resnik, Parsons Infrastructure and Technology Group, Inc., USA, 2001.

ZHANG, L. L.; WANG, J. X.; XIANG, Y.; ZENG, X. F.; CHEN, J. F. Absorption of Carbon Dioxide with Ionic Liquid in a Rotating Packed Bed Contactor: Mass Transfer Study. Ind. Eng. Chem. Res. v. 50, p. 6957, 2011. 